\title{
Stability Analysis of the Supercritical Surface Quasi-Geostrophic Equation
}

\author{
Yan Jia, Xingguo Gui, and Bo-Qing Dong \\ School of Mathematical Sciences, Anhui University, Hefei 230601, China \\ Correspondence should be addressed to Bo-Qing Dong; bqdong@ahu.edu.cn
}

Received 9 June 2013; Accepted 21 August 2013

Academic Editor: Diego Córdoba

Copyright (C) 2013 Yan Jia et al. This is an open access article distributed under the Creative Commons Attribution License, which permits unrestricted use, distribution, and reproduction in any medium, provided the original work is properly cited.

\begin{abstract}
This paper is devoted to the study of the stability issue of the supercritical dissipative surface quasi-geostrophic equation with nondecay low-regular external force. Supposing that the weak solution $\theta(x, t)$ of the surface quasi-geostrophic equation with the force $f \in L^{2}\left(0, T ; H^{-\alpha / 2}\left(\mathbb{R}^{2}\right)\right)$ satisfies the growth condition in the critical BMO space $\nabla \theta \in L^{1}(0, \infty$; BMO), it is proved that every perturbed weak solution $\bar{\theta}(t)$ converges asymptotically to solution $\theta(t)$ of the original surface quasi-geostrophic equation. The initial and external forcing perturbations are allowed to be large.
\end{abstract}

\section{Introduction and Main Results}

Mathematical models in fluid dynamics play an important role in theoretical and computational studies in meteorological and oceanographic sciences and petroleum industries, and so forth. In this paper, we consider a simple mathematical model of large scalar ocean and atmosphere dynamics (see Pedlosky [1]) — the dissipative surface quasi-geostrophic equation which is first introduced by Constantin et al. [2]:

$$
\begin{gathered}
\frac{\partial \theta}{\partial t}+u \cdot \nabla \theta+\kappa \Lambda^{\alpha} \theta=f \\
(x, t) \in \mathbb{R}^{2} \times(0, \infty), \\
\theta(x, 0)=\theta_{0}, \quad x \in \mathbb{R}^{2} .
\end{gathered}
$$

Here $\kappa>0$ is a dissipative coefficient and $\Lambda$ is the Riesz potential operator defined by the fractional power of $-\Delta$ :

$$
\Lambda=(-\Delta)^{1 / 2}, \quad \widehat{\Lambda^{\alpha} g}=\left(\widehat{-\Delta)^{\alpha / 2}} g=|\xi|^{\alpha} \widehat{g} .\right.
$$

$\theta(x, t)$ is an unknown scalar function representing potential temperature. $u(x, t)$ is the velocity field determined by

$$
u=-\nabla^{\perp}(-\Delta)^{-1 / 2} \theta=-\mathscr{R}^{\perp} \theta=\left(\mathscr{R}_{2} \theta,-\mathscr{R}_{1} \theta\right),
$$

where $\mathscr{R}_{j}, j=1,2$ is the $2 \mathrm{D}$ Riesz transform, $\theta_{0}(x)$ is the initial data, and $f(x, t)$ is the nondecay external force.
The surface quasi-geostrophic equation shares many features with fundamental fluid motion equations. When $\kappa=0$, it is comparable to the formation of sharp fronts between masses of hot and cold air (see Majda and Tabak [3]). When $\alpha=0$ (1) can be looked to as a nonviscous wind driven circulation equation (see Pedlosky [1]). What is more, (1) with $\alpha=1$ shares many similar features with three-dimensional incompressible Navier-Stokes equations [4]. Thus $\alpha=1$ is therefore referred to as the critical case, while the cases $0<$ $\alpha<1$ and $1<\alpha \leq 2$ are supercritical and subcritical, respectively.

Due to the importance in mathematics, there is much attention on the well-posedness and large time behaviors of the surface quasi-geostrophic equation. When $1<\alpha \leq 2$, Constantin and $\mathrm{Wu}[5]$ proved that this equation possesses a unique and global smooth solution for a sufficiently smooth initial function. When $\alpha=1$, Kiselev et al. [6] showed the existence of global smooth solutions for smooth periodic initial data. At the same time, Caffarelli and Vasseur [7] also constructed a global smooth solution with $\theta_{0} \in L^{2}\left(\mathbb{R}^{n}\right)$. In the supercritical case $0<\alpha<1$, however, the question of the global regularity is still generally not clear although many efforts have been made on the small regular solution or regularity criteria (see [8-16]). One may also refer to some interesting results of the generalized quasi-geostrophic equations (see [17-21]). 
However, it is desirable to understand the asymptotic stability issue of the supercritical dissipative surface quasigeostrophic equation. More precisely, we consider the perturbed quasi-geostrophic equation:

$$
\begin{gathered}
\frac{\partial \bar{\theta}}{\partial t}+\bar{u} \cdot \nabla \bar{\theta}+\kappa \Lambda^{\alpha} \bar{\theta}=f+g, \\
(x, t) \in \mathbb{R}^{2} \times(0, \infty), \\
\bar{\theta}(x, 0)=\bar{\theta}_{0}=\theta_{0}+w_{0}, \quad x \in \mathbb{R}^{2},
\end{gathered}
$$

where $w_{0}(x)$ and $g(x, t)$ are the initial data and external forcing perturbations. The stability problem of (4) has been studied by many authors and a lot of good results have been obtained. To go directly to the main points of the present paper, in what follows we only review some former results which are closely related to our main results. First, when the surface quasi-geostrophic equations (1) has zero external force or time-decay external force, some interesting time decay results [22-25] have been obtained which essentially show the asymptotic stability for the trivial solution $\theta(x, t)=$ 0 . For the stability issue of the nontrivial solutions, Chae and Lee [26] studied the asymptotic stability of critical quasigeostrophic equation in Besov space under the small initial perturbation. The nonlinear stability and instability analysis of the subcritical quasi-geostrophic equation is recently investigated by Chen and Price [27]. Dong and Chen [28] recently considered the asymptotic stability problem in the Serrin-type critical Lebesgue space $L^{p}\left(\mathbb{R}^{2}\right)(p<\infty)$ under the $L^{2}\left(\mathbb{R}^{2}\right)$ external force. It is natural and interesting to further investigate the asymptotic stability for the nontrivial global solutions in the larger critical spaces, especially in the margin case $p=\infty$.

The main purpose of this study is to investigate the asymptotic stability for the global solution of the supercritical surface quasi-geostrophic equation in the critical BMO space with nondecay low-regular external force. To state our main results, we first give the definition of the weak solution of the dissipative surface quasi-geostrophic equation (1).

Definition 1. Letting $\theta_{0} \in L^{2}\left(\mathbb{R}^{2}\right), f \in L^{2}\left(0, T ; H^{-\alpha / 2}\left(\mathbb{R}^{2}\right)\right)$. A measurable function $\theta(x, t)$ is said to be a global weak solution of the surface quasi-geostrophic equation (1) if the following conditions hold true.

(i) $\theta(x, t) \in L^{\infty}\left(0, T ; L^{2}\left(\mathbb{R}^{2}\right)\right) \cap L^{2}\left(0, T ; H^{\alpha / 2}\left(\mathbb{R}^{2}\right)\right)$ for all $T>0$.

(ii) $\theta(x, t)$ is continuous on $[0, \infty)$ in the weak topology of $L^{2}\left(\mathbb{R}^{2}\right)$ and $(\theta(t), \phi) \rightarrow\left(\theta_{0}, \phi\right)$ as $t \rightarrow 0$ for all $\phi \in L^{2}\left(\mathbb{R}^{2}\right)$.

(iii) For $0 \leq s \leq t \leq \infty$, and any test function $\varphi \in$ $C^{1}\left([s, t] ; H^{\alpha / 2}\left(\mathbb{R}^{2}\right)\right)$

$(\theta(t), \varphi(t))$

$$
\begin{aligned}
& +\int_{s}^{t}\left\{-\left(\theta, \partial_{\tau} \varphi\right)+\kappa\left(\Lambda^{\alpha / 2} \theta, \Lambda^{\alpha / 2} \varphi\right)-(\theta, u \cdot \nabla \varphi)\right\} d \tau \\
= & (\theta(s), \varphi(s))+\int_{s}^{t}(f, \varphi) d \tau,
\end{aligned}
$$

where $(\cdot, \cdot)$ denotes the inner product of the space $L^{2}\left(\mathbb{R}^{2}\right)$.

(iv) Energy-type inequality

$$
\begin{aligned}
& \|\theta(t)\|_{L^{2}}^{2}+2 \kappa \int_{s}^{t}\left\|\Lambda^{\alpha / 2} \theta(\tau)\right\|_{L^{2}}^{2} d \tau \\
& \quad \leq\|\theta(s)\|_{L^{2}}^{2}+2 \int_{s}^{t}(f, \theta) d \tau \quad 0 \leq s \leq t \leq \infty .
\end{aligned}
$$

Now our results read as the following.

Theorem 2. Letting $0<\alpha<1, \theta_{0} \in L^{2}\left(\mathbb{R}^{2}\right)$. Assume $\theta(x, t)$ is a global weak solution of the supercritical dissipative surface quasi-geostrophic equation (1) with the nondecay low-regular external force $f \in L^{2}\left(0, T ; H^{-\alpha / 2}\left(\mathbb{R}^{2}\right)\right)$ and satisfies the critical growth condition in $B M O$ space:

$$
\nabla \theta \in L^{1}(0, \infty ; B M O) .
$$

Then, for any perturbations $w_{0} \in L^{2}\left(\mathbb{R}^{2}\right)$ and $g \in L^{2}(0, \infty$; $\left.H^{-\alpha / 2}\left(\mathbb{R}^{2}\right)\right)$, every weak solution $\bar{\theta}(x, t)$ of the perturbed quasigeostrophic equation (4) converges asymptotically to solution $\theta(t)$ as

$$
\|\bar{\theta}(t)-\theta(t)\|_{L^{2}} \longrightarrow 0 \quad(t \longrightarrow \infty) .
$$

Remark 3. The critical space-time mixed space (7) in Theorem 2 is scaling invariant under the scaling transformation $\theta_{\lambda}(x, t)=\lambda^{\alpha-1} \theta\left(\lambda x, \lambda^{\alpha} t\right)$. Moreover, according to the result of Dong and Chen [29], the weak solution $\theta(x, t)$ satisfying (7) is actually regular. Furthermore, our results also show that the weak solutions $\bar{\theta}(x, t)$ of the perturbed equation (4) do not need to satisfy the energy-type inequality (6) and then they do not have to be regular.

Remark 4. On one hand, compared with the stability result by Chae and Lee [26], there is no any smallness restriction on the perturbation functions $w_{0}$ and $g$ in Theorem 2 . That is to say, the initial and external forcing perturbations from $\theta(t)$ are allowed to be large. On the other hand, our result here extends the previous result [28] to the margin case $p=\infty$ due to the embedding relation $L^{\infty} \varsubsetneqq$ BMO. Moreover, in comparison with [28], we require less regularity for the external force.

Remark 5. Although Theorem 2 shows that the decay property of difference between the global solution and the perturbed solution $\|\bar{\theta}(t)-\theta(t)\|_{L^{2}} \rightarrow 0$, however, it is different from the time decay issue of the zero-forced surface quasigeostrophic equations (see $[22,23]$ and references therein) $\|\theta(t)\|_{L^{2}} \rightarrow 0$. The latter indeed shows the asymptotic stability of the trivial solution $\theta=0$ for the surface quasigeostrophic equation with zero external force. However, when the surface quasi-geostrophic equation (1) has the nonzero low-regular external force $f \in L^{2}\left(0, T ; H^{-\alpha / 2}\left(\mathbb{R}^{2}\right)\right)$, $\theta=0$ is obviously not a solution of (1), therefore, our result here essentially shows the asymptotic stability of the nonzero global solution of (1). 
Remark 6. The main idea in the proof of Theorem 2 is based on some new observations due to the special structure in nonlinear term $u \cdot \nabla \theta$ which is different from the classic Navier-Stokes equations. Additionally, in order to derive an auxiliary decay estimate in the framework of the critical BMO space, we also need to choose a suitable test function and derive some average decay properties. It is worth noting that although the study of the classic incompressible NavierStokes equations is beneficial to that of quasi-geostrophic equation, compared with some asymptotic stability results of three-dimensional Navier-Stokes equations (refer to [3032]), we do not know, however, whether our result in the framework of the critical BMO space is still valid for the Navier-Stokes equations. We will focus on that problem in the future.

The remains of this paper are organized as follows. In Section 2, we will investigate the global $L^{2}$ estimates of the difference between the original surface quasi-geostrophic equation and the perturbed equation. In Section 3, we will further derive an average decay estimates of the difference. Finally, we will prove the asymptotic stability of large solution to the surface quasi-geostrophic equation (1) under the large initial perturbation in Section 4.

Let us end this section by some notations. In what follows, $C$ stands for the abstractly positive constant. $L^{p}\left(\mathbb{R}^{2}\right)$ with $1 \leq$ $p \leq \infty$ denotes the usual Lebesgue space. $H^{s}\left(\mathbb{R}^{2}\right)$ with $s \in \mathbb{R}$ is the fractional Sobolev space with the norm:

$$
\|f\|_{H^{s}}=\left(\int_{\mathbb{R}^{2}}|\xi|^{2 s}|\widehat{f}|^{2} d \xi\right)^{1 / 2} .
$$

BMO is the space of the bounded mean oscillation defined by

$$
\begin{aligned}
\mathrm{BMO}= & \left\{f \in L_{\mathrm{loc}}^{1}\left(\mathbb{R}^{2}\right) ; \sup _{x, r} \frac{1}{\left|B_{r}(x)\right|}\right. \\
& \left.\times \int_{B_{r}(x)}\left|f(y)-\bar{f}_{B_{r}(x)}\right| d y<\infty\right\},
\end{aligned}
$$

where

$$
\bar{f}_{B_{r}(x)}=\frac{1}{\left|B_{r}(x)\right|} \int_{B_{r}(x)} f(y) d y
$$

is the average of $f$ over $B_{r}(x)$. In particular, BMO is the dual space of the Hardy space $\mathscr{H}^{1}$.

\section{Global Estimate of $\theta-\bar{\theta}$}

In this section, we will investigate the global $L^{2}$ estimates of the difference $w=\theta-\bar{\theta}$ between the global solution $\theta$ of the original equation (1) and the weak solution $\bar{\theta}$ of the perturbed equation (4). It is mentioned that some necessary smooth properties of the difference $w$ is required in the derivation of the global $L^{2}$ estimates below. As stated in Remark 3, the global weak solution $\theta$ of the original equation (1) satisfying the regular condition (6) is actually regular. However, the perturbed weak solution $\bar{\theta}$ is not smooth, in order to derive the global estimates of the difference $w=\theta-\bar{\theta}$. We first seek a smooth function to approximate the weak solution $\bar{\theta}$ of the perturbed quasi-geostrophic equation (4). To do so, we apply the standard Galerkin method to construct the smooth approximate solutions:

$$
\bar{\theta}_{m}(x, t)=\sum_{k=1}^{m} g_{k m}(t) \phi_{k}(x), \quad m \in N
$$

Here $\left\{\phi_{k}\right\}_{k=1}^{\infty} \in C^{\infty}\left(\mathbb{R}^{2}\right)$ is an orthonormal basis of $L^{2}\left(\mathbb{R}^{2}\right)$.

Instead of the perturbed quasi-geostrophic equation (4), for any test function $\phi \in X_{m}=$ the space spanned by $\phi_{1}, \phi_{2}, \ldots, \phi_{m}, \bar{\theta}_{m}(x, t)$ satisfy

$$
\begin{gathered}
\left(\frac{\partial \bar{\theta}_{m}}{\partial t}+\bar{u}_{m} \cdot \nabla \bar{\theta}_{m}+\kappa \Lambda^{\alpha} \bar{\theta}_{m}, \phi\right)=\left(f_{m}+g_{m}, \phi\right), \\
\bar{\theta}_{m}(x, 0)=\sum_{k=1}^{m}\left(\bar{\theta}_{0}, \phi_{k}\right) \phi_{k},
\end{gathered}
$$

where

$$
f_{m}=\sum_{k=1}^{m}\left(f, \phi_{k}\right) \phi_{k}, \quad g_{m}=\sum_{k=1}^{m}\left(g, \phi_{k}\right) \phi_{k} .
$$

It is worth noting that, for fixed integer $m$, equations (13) are indeed ordinary differential equations. The theory of existence and uniqueness for such ordinary differential equations is standard. Therefore, we can prove that the approximated solutions $\bar{\theta}_{m}$ exist globally and smoothly and are uniformly bounded in the following space:

$$
\bar{\theta}_{m} \in L^{\infty}\left(0, T ; L^{2}\left(\mathbb{R}^{2}\right)\right) \cap L^{2}\left(0, T ; H^{\alpha / 2}\left(\mathbb{R}^{2}\right)\right), \quad \forall T>0 .
$$

Furthermore, it allows us to prove that the approximates smooth solutions $\bar{\theta}_{m}$ converge to the weak solution of the perturbed quasi-geostrophic equation (4) when $m$ tends to infinity. Indeed, we only need to obtain the strong convergence of $\bar{\theta}_{m}$ in space $L_{\text {loc }}^{2}\left(\mathbb{R}^{2} \times(0, \infty)\right)$. To do so, for example, with the aid of Hölder and Sobolev embedding estimates, it follows that

$$
\begin{aligned}
\left|\left(\bar{u}_{m} \cdot \nabla \bar{\theta}_{m}, \phi\right)\right| & \leq C\left\|\bar{u}_{m}\right\|_{L^{2}}\left\|\bar{\theta}_{m}\right\|_{L^{4 /(2-\alpha)}}\|\nabla \phi\|_{L^{4 / \alpha}} \\
& \leq C\left\|\bar{u}_{m}\right\|_{L^{2}}\left\|\bar{\theta}_{m}\right\|_{H^{\alpha / 2}}\|\phi\|_{H^{(4-\alpha) / 2}}
\end{aligned}
$$

for $\phi \in H^{(4-\alpha) / 2}$. This implies the uniform bounds of

$$
\begin{gathered}
\frac{\partial \bar{\theta}_{m}}{\partial t}=-\bar{u}_{m} \cdot \nabla \bar{\theta}_{m}-k \Lambda^{\alpha} \bar{\theta}_{m}+f_{m}+g_{m} \\
\text { in the space } L^{2}\left(0, T ; H^{-(4-\alpha) / 2}\left(\mathbb{R}^{2}\right)\right)
\end{gathered}
$$

By the compactness theorem [4, Chapter 3, Theorem 2.1], the sequence $\bar{\theta}_{m}$ admits a subsequence denoted also by $\bar{\theta}_{m}$ 
converging to the weak solution of the perturbed equation (4):

$$
\bar{\theta}_{m} \longrightarrow \bar{\theta} \quad(m \longrightarrow \infty) \text { strongly in } L_{\text {loc }}^{2}\left(\mathbb{R}^{2} \times(0, \infty)\right),
$$

from which and uniformly bounds of $\left\|\bar{\theta}_{m}(t)\right\|_{L^{2}}$ due to (15), we have

$$
\bar{\theta}_{m}(t) \longrightarrow \bar{\theta}(t) \quad(m \longrightarrow \infty) \text { weakly in } L^{2}\left(\mathbb{R}^{2}\right)
$$

for almost all $t \in(0, \infty)$.

Thanks to the regular solution $\theta \in L^{\infty}\left(0, T ; L^{2}\left(\mathbb{R}^{2}\right)\right)$, we then derive from (19) that

$$
\begin{array}{r}
\theta(t)-\bar{\theta}_{m}(t) \longrightarrow \theta(t)-\bar{\theta}(t) \\
(m \longrightarrow \infty) \text { weakly in } L^{2}\left(\mathbb{R}^{2}\right)
\end{array}
$$

for almost all $t \in(0, \infty)$, which implies by Fatou Lemma that

$$
\|\theta(t)-\bar{\theta}(t)\|_{L^{2}} \leq \liminf _{m \rightarrow \infty}\left\|\theta(t)-\bar{\theta}_{m}(t)\right\|_{L^{2}}
$$

for almost all $t \in(0, \infty)$.

Denoting by $w_{m}=\theta-\bar{\theta}_{m}$ the difference between solution $\theta$ of original equation (1) and approximated smooth solution $\bar{\theta}_{m}$ of the (13), with the direct computation due to the smoothness of $w_{m}$, it is easy to examine $w_{m}$ satisfying

$$
\begin{aligned}
&\left\|w_{m}(t)\right\|_{L^{2}}^{2}+2 \kappa \int_{0}^{t}\left\|\Lambda^{\alpha / 2} w_{m}\right\|_{L^{2}}^{2} d \tau \\
& \leq\left\|\theta_{0}-\bar{\theta}_{m}(0)\right\|_{L^{2}}^{2} \\
&+2 \int_{0}^{t}\left\{\left(u \cdot \nabla \theta, \bar{\theta}_{m}\right)-\left(\bar{u}_{m} \cdot \nabla \bar{\theta}_{m}, \theta\right)\right\} d \tau \\
&+2 \int_{0}^{t}\left(f_{m}+g_{m}-f, w_{m}\right) d \tau \\
& \leq\left\|\theta_{0}-\bar{\theta}_{m}(0)\right\|_{L^{2}}^{2}-2 \int_{0}^{t}\left(\left(u-\bar{u}_{m}\right) \cdot \nabla \theta, w_{m}\right) d \tau \\
&+2 \int_{0}^{t}\left(f_{m}+g_{m}-f, w_{m}\right) d \tau,
\end{aligned}
$$

where the divergence free property of the velocity is used. In order to deal with the nonlinear term of the right hand side of (22), we require the following important lemma.

Lemma 7 (Marchand [33]). Assume that $h \in L^{2}\left(\mathbb{R}^{2}\right), \mathscr{R}_{i}(i \in$ $\{1,2\})$ is the Riesz transform, then there exists a constant $C$ such that

$$
\left\|h \mathscr{R}_{i} h\right\|_{\mathscr{H}^{1}} \leq C\|h\|_{L^{2}}^{2}
$$

is valid. Here $\mathscr{H}^{1}$ is the Hardy space.
Thus we have the following crucial estimates for the nonlinear term of (23):

$$
\begin{aligned}
& \left|-2 \int_{0}^{t}\left(\left(u-\bar{u}_{m}\right) \cdot \nabla \theta, w_{m}\right) d \tau\right| \\
& \quad \leq C\left|\int_{0}^{t} \int_{\mathbb{R}^{2}} \mathscr{R}^{\perp} w_{m} \cdot \nabla \theta w_{m} d x d s\right| \\
& \quad \leq C \int_{0}^{t}\left(\left\|w_{m} \mathscr{R}_{1} w_{m}\right\|_{\mathscr{H}^{1}}+\left\|w_{m} \mathscr{R}_{2} w_{m}\right\|_{\mathscr{C}^{1}}\right)\|\nabla \theta\|_{\mathrm{BMO}} d s \\
& \quad \leq C \int_{0}^{t}\left\|w_{m}\right\|_{L^{2}}^{2}\|\nabla \theta\|_{\mathrm{BMO}} d s,
\end{aligned}
$$

where we have also used the fact that BMO is the dual space of the Hardy space $\mathscr{H}^{1}$.

With the aid of the Hölder inequality and the Young inequality, one shows that for the external force term

$$
\begin{aligned}
\mid 2 \int_{0}^{t}( & \left.f_{m}+g_{m}-f, w_{m}\right) d \tau \mid \\
\leq & C \int_{0}^{t}\left\|g_{m}\right\|_{H^{-\alpha / 2}}\left\|w_{m}\right\|_{H^{\alpha / 2}} d \tau \\
& +C \int_{0}^{t}\left\|f_{m}-f\right\|_{H^{-\alpha / 2}}\left\|w_{m}\right\|_{H^{\alpha / 2}} d \tau \\
\leq & C \int_{0}^{t}\left(\left\|g_{m}\right\|_{H^{-\alpha / 2}}^{2}+\left\|f_{m}-f\right\|_{H^{-\alpha / 2}}^{2}\right) d \tau \\
& +\kappa \int_{0}^{t}\left\|w_{m}\right\|_{H^{\alpha / 2}}^{2} d \tau .
\end{aligned}
$$

Plugging the above inequalities into (22) and taking the Gronwall inequality into consideration, we derive the global $L^{2}$ estimate of $w_{m}$ :

$$
\begin{aligned}
& \left\|w_{m}(t)\right\|_{L^{2}}^{2}+\kappa \int_{0}^{t}\left\|\Lambda^{\alpha / 2} w_{m}(\tau)\right\|_{L^{2}}^{2} d \tau \\
& \leq\left(\left\|\theta_{0}-\bar{\theta}_{m}(0)\right\|_{L^{2}}^{2}\right. \\
& \left.\quad+C \int_{0}^{t}\left(\left\|g_{m}\right\|_{H^{-\alpha / 2}}^{2}+\left\|f_{m}-f\right\|_{H^{-\alpha / 2}}^{2}\right) d \tau\right) \\
& \quad \times \exp \left\{C \int_{0}^{t}\|\nabla \theta\|_{\mathrm{BMO}} d \tau\right\} .
\end{aligned}
$$

Passing the limit $m \rightarrow \infty$ in the above inequality and applying (21), we have 


$$
\begin{aligned}
&\|w(t)\|_{L^{2}}^{2}+\kappa \int_{0}^{t}\left\|\Lambda^{\alpha / 2} w(\tau)\right\|_{L^{2}}^{2} d \tau \\
& \leq \liminf _{m \rightarrow \infty}\left\{\left\|w_{m}(t)\right\|_{L^{2}}^{2}+\kappa \int_{0}^{t}\left\|\Lambda^{\alpha / 2} w_{m}(\tau)\right\|_{L^{2}}^{2} d \tau\right\} \\
& \leq\left(\left\|w_{0}\right\|_{L^{2}}^{2}+C \int_{0}^{t}\|g\|_{H^{-\alpha / 2}}^{2} d \tau\right) \exp \left\{C \int_{0}^{t}\|\nabla \theta\|_{\mathrm{BMO}} d \tau\right\} \\
& \leq\left(\left\|w_{0}\right\|_{L^{2}}^{2}+C \int_{0}^{\infty}\|g\|_{H^{-\alpha / 2}}^{2} d \tau\right) \\
& \times \exp \left\{C \int_{0}^{\infty}\|\nabla \theta\|_{\mathrm{BMO}} d \tau\right\} \\
&=: E_{0}<\infty
\end{aligned}
$$

for the constant $E_{0}$ independent of $t>0$.

Moreover, by the slight modification of the derivation of (22) and (27), it is not difficult to derive the following global estimates of $w$ :

$$
\begin{aligned}
& \|w(t)\|_{L^{2}}^{2}+\kappa \int_{s}^{t}\left\|\Lambda^{\alpha / 2} w\right\|_{L^{2}}^{2} d \tau \\
& \quad \leq\|w(s)\|_{L^{2}}^{2}+C \int_{s}^{t}\|g\|_{H^{-\alpha / 2}}^{2} d \tau+C \int_{s}^{t}\|\nabla \theta\|_{\mathrm{BMO}} d \tau
\end{aligned}
$$

for $0 \leq s \leq t \leq \infty$.

\section{Average Decay of $\theta-\bar{\theta}$}

In this section, we are devoted to investigating the average decay of the difference $w=\bar{\theta}-\theta$ which will play an important role in the proof of Theorem 2.

It is worth noting that Kozono [30] and Zhou [32] have investigated the average decay properties of the classic Navier-Stokes equation. For the surface quasi-geostrophic equation considered here, however, the fractional dissipative operators $\Lambda^{\alpha}=(-\Delta)^{\alpha / 2}$ are nonlocal singular integral operators. the new difficulty on the uniformly estimates of $w$ will rise if we directly follow the similar argument of the classic Navier-Stokes equations [30, 32].

Fortunately, we can avoid those additional difficulties with the aid of the theory of the analytic semigroup (see Pazy [34]). Since the Laplacian operator $-\Delta$ in whole space $\mathbb{R}^{2}$ generates a bounded analytic semigroup in each $L^{p}\left(\mathbb{R}^{2}\right)$ $(1<p<\infty)$, thus the fractional power $\Lambda^{\alpha}=(-\Delta)^{\alpha / 2}$ can be redefined with the aid of the spectral decomposition:

$$
\Lambda^{\alpha}=\int_{0}^{\infty} \lambda^{\alpha / 2} d E(\lambda)
$$

where $E(\lambda)$ is a family of projection operators and $\lambda(0<\lambda<$ $\infty)$ is the spectral of the Laplacian operator $-\Delta$. Namely, the analytic semigroup $e^{-t \Lambda^{\alpha}}$ generated by the nonlocal singular integral operators $\Lambda^{\alpha}$ is also defined in a satisfactory form:

$$
e^{-t \Lambda^{\alpha}}=\int_{0}^{\infty} e^{-t \lambda^{\alpha / 2}} d E(\lambda)
$$

Furthermore, we may derive the embedding relation in a natural form

$$
\begin{array}{r}
\|\theta\|_{L^{p}} \leq C\|\theta\|_{H^{\alpha}}=C\left\||\xi|^{\alpha} \hat{\theta}\right\|_{L^{2}}=C\left\|\Lambda^{\alpha} \theta\right\|_{L^{2}}, \\
\text { with } \frac{1}{p}=\frac{1}{2}-\frac{\alpha}{2},
\end{array}
$$

where Parseval equality is used in the last two equalities.

Now we carry out to study the average decay of the difference $w=\theta-\bar{\theta}$. Firstly, according to the definitions of the weak solutions in Definition 1, we choose a special test function $\varphi_{\varepsilon}$ as

$$
\begin{aligned}
\varphi_{\varepsilon}(\tau)=\int_{s}^{t} \eta_{\varepsilon}(\tau-\sigma)\left(1+\Lambda^{2}\right)^{-(3-\alpha) / 2} e^{-\kappa(2 t-\tau-\sigma) \Lambda^{\alpha}} \\
\times w(\sigma) d \sigma, \quad \varepsilon>0,
\end{aligned}
$$

where $\eta_{\varepsilon}(t)=(1 / \varepsilon) \eta(|t / \varepsilon|)>0$ is the standard mollifier function with $\eta(t) \in C_{0}^{\infty}(-1,1)$ and $\int_{-1}^{1} \eta(t) d t=1$. Thus $w(x, t)$ satisfies the following weak formation

$$
\begin{gathered}
\int_{s}^{t}\left(\partial_{\tau} w+\kappa \Lambda^{\alpha} w, \varphi_{\varepsilon}\right) d \tau+\int_{s}^{t}\left(u \cdot \nabla \theta-\bar{u} \cdot \nabla \bar{\theta}, \varphi_{\varepsilon}\right) d \tau \\
+\int_{s}^{t}\left(g, \varphi_{\varepsilon}\right) d \tau=0
\end{gathered}
$$

for $0 \leq s \leq t<\infty$.

We now give the estimates of (33) by passing the limit $\varepsilon \rightarrow 0$. More precisely, we will prove the following lemma.

Lemma 8. Under the same condition in Theorem 2, we have the following uniform estimates of the difference $w$ :

$$
\begin{aligned}
\left\|\left(1+\Lambda^{2}\right)^{-(3-\alpha) / 4} w(t)\right\|_{L^{2}}^{2} \\
-\left\|e^{-\kappa(t-s) \Lambda^{\alpha}}\left(1+\Lambda^{2}\right)^{-(3-\alpha) / 4} w(s)\right\|_{L^{2}}^{2} \\
\leq C \int_{s}^{t}\left\|\Lambda^{\alpha / 2} \theta\right\|_{L^{2}}^{2} d \tau+C \int_{s}^{t}\left\|\Lambda^{\alpha / 2} w\right\|_{L^{2}}^{2} d \tau \\
+C \int_{s}^{t}\|g\|_{H^{-\alpha / 2}}^{2} d \tau .
\end{aligned}
$$

We will give the proof at the end of this section. Once the crucial uniform estimates (34) is obtained, we can obtain the average decay of the difference $w$. In fact, letting $t \rightarrow \infty$ in (34), since

$$
\lim _{t \rightarrow \infty}\left\|e^{-\kappa(t-s) \Lambda^{\alpha}}\left(1+\Lambda^{2}\right)^{-(3-\alpha) / 4} w(s)\right\|_{L^{2}}^{2}=0,
$$

then one shows that for (34)

$$
\begin{aligned}
\limsup _{t \rightarrow \infty}\left\|\left(1+\Lambda^{2}\right)^{-(3-\alpha) / 4} w(t)\right\|_{L^{2}}^{2} \\
\leq C \int_{s}^{\infty}\left\|\Lambda^{\alpha / 2} \theta\right\|_{L^{2}}^{2} d \tau+C \int_{s}^{\infty}\left\|\Lambda^{\alpha / 2} w\right\|_{L^{2}}^{2} d \tau \\
+C \int_{s}^{\infty}\|g\|_{H^{-\alpha / 2}}^{2} d \tau,
\end{aligned}
$$


and then letting $s \rightarrow \infty$, we have

$$
\limsup _{t \rightarrow \infty}\left\|\left(1+\Lambda^{2}\right)^{-(3-\alpha) / 4} w(t)\right\|_{L^{2}}^{2}=0
$$

Furthermore, employing the Parseval equality and the Hölder inequality, we obtain that for $\|w\|_{L^{2}}$

$$
\begin{aligned}
\|w\|_{L^{2}}^{2}= & C \int_{\mathbb{R}^{2}}|\widehat{w}|^{2} d \xi \\
= & C \int_{\mathbb{R}^{2}}\left(1+|\xi|^{2}\right)^{\left(\alpha^{2}-3 \alpha\right) / 6}|\widehat{w}|^{2 \alpha / 3} \\
& \times\left(1+|\xi|^{2}\right)^{\left(3 \alpha-\alpha^{2}\right) / 6}|\widehat{w}|^{(6-2 \alpha) / 3} d \xi \\
\leq & C\left(\int_{\mathbb{R}^{2}}\left(1+|\xi|^{2}\right)^{-(3-\alpha) / 2}|\widehat{w}|^{2} d \xi\right)^{\alpha / 3} \\
& \times\left(\int_{\mathbb{R}^{2}}\left(1+|\xi|^{2}\right)^{\alpha / 2}|\widehat{w}|^{2} d \xi\right)^{(3-\alpha) / 3} \\
\leq & C\left(\int_{\mathbb{R}^{2}}\left(1+|\xi|^{2}\right)^{-(3-\alpha) / 2}|\widehat{w}|^{2} d \xi\right)^{\alpha / 3} \\
& \times\left(\int_{\mathbb{R}^{2}}\left(|\widehat{w}|^{2}+|\xi|^{\alpha}|\widehat{w}|^{2}\right) d \xi\right)^{(3-\alpha) / 3} \\
\leq & C\left\|\left(1+\Lambda^{2}\right)^{-(3-\alpha) / 4} w\right\|_{L^{2}}^{2 \alpha / 3} \\
& \times\left(\|w\|_{L^{2}}^{2}+\left\|\Lambda^{\alpha / 2} w\right\|_{L^{2}}^{2}\right)^{1-(\alpha / 3)} \cdot
\end{aligned}
$$

Integrating in time from $t / 2$ to $t$ yields

$$
\begin{aligned}
& \frac{1}{t} \int_{t / 2}^{t}\|w(\tau)\|_{L^{2}}^{2} d \tau \\
& \leq \frac{C}{t} \int_{t / 2}^{t}\left\|\left(1+\Lambda^{2}\right)^{-(3-\alpha) / 4} w\right\|_{L^{2}}^{2 \alpha / 3} \\
& \quad \times\left(\|w\|_{L^{2}}^{2}+\left\|\Lambda^{\alpha / 2} w\right\|_{L^{2}}^{2}\right)^{1-(\alpha / 3)} d \tau \\
& \leq C\left(\frac{1}{t} \int_{t / 2}^{t}\left\|\left(1+\Lambda^{2}\right)^{-(3-\alpha) / 4} w\right\|_{L^{2}}^{2} d \tau\right)^{\alpha / 3} \\
& \quad \times\left(\frac{1}{t} \int_{t / 2}^{t}\left(\|w\|_{L^{2}}^{2}+\left\|\Lambda^{\alpha / 2} w\right\|_{L^{2}}^{2}\right) d \tau\right)^{1-(\alpha / 3)} \\
& \leq C\left(\frac{1}{t} \int_{t / 2}^{t}\left\|\left(1+\Lambda^{2}\right)^{-(3-\alpha) / 4} w\right\|_{L^{2}}^{2} d \tau\right)^{\alpha / 3}\left(1+\frac{1}{t}\right)^{1-(\alpha / 3)} \\
& \leq C \lim \sup \left\|\left(1+\Lambda^{2}\right)^{-(3-\alpha) / 4} w(\tau)\right\|_{L^{2}}^{2}
\end{aligned}
$$

Together with (37) we derive the desired average decay of the difference $w=\theta-\bar{\theta}$ between the global solution $\theta$ of the original quasi-geostrophic equation (1) and the weak solution $\bar{\theta}$ of the perturbed quasi-geostrophic equation (4):

$$
\lim _{t \rightarrow \infty} \frac{1}{t} \int_{t / 2}^{t}\|w(\tau)\|_{L^{2}}^{2} d \tau=0
$$

Now it remains to prove Lemma 8.

Proof of Lemma 8. For convenience, the three terms of the left hand side of (33) are denoted by $I, J$, and $K$, respectively. For $I$, we have

$$
\begin{aligned}
I= & \int_{s}^{t}\left(\partial_{\tau} w+\kappa \Lambda^{\alpha} w, \varphi_{\varepsilon}\right) d \tau \\
= & \int_{s}^{t} \frac{d}{d \tau}\left(\int_{\mathbb{R}^{2}} w \varphi_{\varepsilon} d x\right) d \tau \\
& +\int_{s}^{t} \int_{\mathbb{R}^{2}}\left(-w \partial_{\tau} \varphi_{\varepsilon}+\kappa w \Lambda^{\alpha} \varphi_{\varepsilon}\right) d x d \tau \\
= & \int_{\mathbb{R}^{2}}\left(w \varphi_{\varepsilon}\right)(t) d x-\int_{\mathbb{R}^{2}}\left(w \varphi_{\varepsilon}\right)(s) d x \\
& +\int_{s}^{t} \int_{\mathbb{R}^{2}} w(\tau)\left(-\partial_{\tau} \varphi_{\varepsilon}+\kappa \Lambda^{\alpha} \varphi_{\varepsilon}\right)(\tau) d x d \tau \\
= & \int_{\mathbb{R}^{2}}\left(w \varphi_{\varepsilon}\right)(t) d x-\int_{\mathbb{R}^{2}}\left(w \varphi_{\varepsilon}\right)(s) d x \\
& -\int_{s}^{t} \int_{\mathbb{R}^{2}} \int_{s}^{t} \partial_{\tau} \eta_{\varepsilon}(\tau-\sigma)\left(1+\Lambda^{2}\right)^{-(3-\alpha) / 2} \\
= & I_{1}+I_{2} . \quad \times e^{-\kappa(2 t-\tau-\sigma) \Lambda^{\alpha}} w(\sigma) w(\tau) d \sigma d x d \tau
\end{aligned}
$$

Applying the Fubini theorem, it is not difficult to obtain

$$
\begin{aligned}
\lim _{\varepsilon \rightarrow 0} \int_{\mathbb{R}^{2}}\left(w \varphi_{\varepsilon}\right)(t) d x & \\
= & \lim _{\varepsilon \rightarrow 0} \int_{\mathbb{R}^{2}} \int_{s}^{t} \eta_{\varepsilon}(t-\sigma)\left(1+\Lambda^{2}\right)^{-(3-\alpha) / 2} e^{-\kappa(t-\sigma) \Lambda^{\alpha}} \\
& \times w(\sigma) w(t) d \sigma d x \\
= & \frac{1}{2} \int_{\mathbb{R}^{2}}\left(1+\Lambda^{2}\right)^{-(3-\alpha) / 2} w(t) w(t) d x \\
= & \frac{1}{2}\left\|\left(1+\Lambda^{2}\right)^{-(3-\alpha) / 4} w(t)\right\|_{L^{2}}^{2}
\end{aligned}
$$

and similarly

$$
\lim _{\varepsilon \rightarrow 0} \int_{\mathbb{R}^{2}}\left(w \varphi_{\varepsilon}\right)(s) d x=\frac{1}{2}\left\|e^{-\kappa(t-s) \Lambda^{\alpha}}\left(1+\Lambda^{2}\right)^{-(3-\alpha) / 4} w(s)\right\|_{L^{2}}^{2},
$$

thus we have for $I_{1}$

$$
\begin{aligned}
\lim _{\varepsilon \rightarrow 0} I_{1}= & \frac{1}{2}\left\|\left(1+\Lambda^{2}\right)^{-(3-\alpha) / 4} w(t)\right\|_{L^{2}}^{2} \\
& -\frac{1}{2}\left\|e^{-\kappa(t-s) \Lambda^{\alpha}}\left(1+\Lambda^{2}\right)^{-(3-\alpha) / 4} w(s)\right\|_{L^{2}}^{2} .
\end{aligned}
$$


For $I_{2}$ we have

$$
\begin{aligned}
I_{2}=-\int_{s}^{t} \int_{\mathbb{R}^{2}} \int_{s}^{t} \partial_{\tau} \eta_{\varepsilon}(\tau-\sigma)\left(1+\Lambda^{2}\right)^{-(3-\alpha) / 2} e^{-\kappa(2 t-\tau-\sigma) \Lambda^{\alpha}} \\
\times w(\sigma) w(\tau) d \sigma d x d \tau \\
=-\frac{1}{\varepsilon} \int_{s}^{t} \int_{\mathbb{R}^{2}} \int_{s}^{t} \partial_{\tau} \eta\left(\left|\frac{\tau-\sigma}{\varepsilon}\right|\right)\left(1+\Lambda^{2}\right)^{-(3-\alpha) / 2} \\
=\frac{1}{\varepsilon} \int_{s}^{t} \int_{\mathbb{R}^{2}} \int_{s}^{t} \partial_{\sigma} \eta\left(\left|\frac{\tau-\sigma}{\varepsilon}\right|\right)\left(1+\Lambda^{2}\right)^{-(3-\alpha) / 2} \\
\quad \times e^{-\kappa(2 t-\tau-\sigma) \Lambda^{\alpha}} w(\sigma) w(\tau) d \sigma d x d \tau \\
=\frac{1}{\varepsilon} \int_{s}^{t} \int_{\mathbb{R}^{2}} \int_{s}^{t} \partial_{\sigma} \eta\left(\left|\frac{\sigma-\tau}{\varepsilon}\right|\right)\left(1+\Lambda^{2}\right)^{-(3-\alpha) / 2} \\
\quad \times e^{-\kappa(2 t-\tau-\sigma) \Lambda^{\alpha}} w(\sigma) w(\tau) d \sigma d x d \tau d \tau \\
=-I_{2}
\end{aligned}
$$

which implies $I_{2}=0$.

Hence, combining the above inequalities, one derives the estimates of $I$ :

$$
\begin{aligned}
\lim _{\varepsilon \rightarrow 0} I= & \frac{1}{2}\left\|\left(1+\Lambda^{2}\right)^{-(3-\alpha) / 4} w(t)\right\|_{L^{2}}^{2} \\
& -\frac{1}{2}\left\|e^{-\kappa(t-s) \Lambda^{\alpha}}\left(1+\Lambda^{2}\right)^{-(3-\alpha) / 4} w(s)\right\|_{L^{2}}^{2} .
\end{aligned}
$$

For $J$, employing integrating by parts gives

$$
\begin{aligned}
J= & \int_{s}^{t}\left(u \cdot \nabla \theta-\bar{u} \cdot \nabla \bar{\theta}, \varphi_{\varepsilon}\right) d \tau \\
= & \int_{s}^{t} \int_{\mathbb{R}^{2}}(\bar{u}-u) \cdot \nabla \varphi_{\varepsilon} w d x d \tau-\int_{s}^{t} \int_{\mathbb{R}^{2}} u \cdot \nabla \varphi_{\varepsilon} w d x d \tau \\
& -\int_{s}^{t} \int_{\mathbb{R}^{2}}(\bar{u}-u) \cdot \nabla \varphi_{\varepsilon} \theta d x d \tau \\
= & : J_{1}+J_{2}+J_{3} .
\end{aligned}
$$

For $J_{1}$, applying the Hölder inequality and the GagliardoNirenberg inequality and (27) gives

$$
\begin{aligned}
J_{1} & =\int_{s}^{t} \int_{\mathbb{R}^{2}}(\bar{u}-u) \cdot \nabla \varphi_{\varepsilon} w d x d \tau \\
& \leq \int_{s}^{t}\left\|\mathscr{R}^{\perp} w\right\|_{L^{8 /(4-\alpha)}}\left\|\nabla \varphi_{\varepsilon}\right\|_{L^{4 / \alpha}}\|w\|_{L^{8 /(4-\alpha)}} d \tau \\
& \leq C \int_{S}^{t}\left\|\nabla \varphi_{\varepsilon}\right\|_{L^{4 / \alpha}}\|w\|_{L^{2}}\left\|\Lambda^{\alpha / 2} w\right\|_{L^{2}} d \tau
\end{aligned}
$$

$$
\begin{aligned}
\leq & C\left(\sup _{0<\tau<\infty}\|w\|_{L^{2}}\right)\left(\int_{s}^{t}\left\|\nabla \varphi_{\varepsilon}\right\|_{L^{4 / \alpha}}^{2} d \tau\right)^{1 / 2} \\
& \times\left(\int_{s}^{t}\left\|\Lambda^{\alpha / 2} w\right\|_{L^{2}}^{2} d \tau\right)^{1 / 2} \\
\leq & E_{0}^{1 / 2} \int_{s}^{t}\left\|\Lambda^{\alpha / 2} w\right\|_{L^{2}}^{2} d \tau,
\end{aligned}
$$

where we have used the following estimates:

$$
\begin{aligned}
& \int_{s}^{t}\left\|\nabla \varphi_{\varepsilon}(\tau)\right\|_{L^{4 / \alpha}}^{2} d \tau \\
& \quad \leq C \int_{s}^{t}\left\|\nabla \varphi_{\varepsilon}(\tau)\right\|_{H^{1-(\alpha / 2)}}^{2} d \tau \\
& \quad=C \int_{s}^{t}\left\||\xi|^{1-(\alpha / 2)} i \xi \widehat{\varphi}_{\varepsilon}(\tau)\right\|_{L^{2}}^{2} d \tau \\
& \quad \leq C \int_{s}^{t}\left\||\xi|^{2-(\alpha / 2)} \widehat{\varphi}_{\varepsilon}(\tau)\right\|_{L^{2}}^{2} d \tau \\
& \quad \leq C \int_{s}^{t} \||\xi|^{2-\alpha} \int_{s}^{t} \eta_{\varepsilon}(\tau-\sigma)\left(1+|\xi|^{2}\right)^{-(3-\alpha) / 2} \\
& \quad \leq C \int_{s}^{t}\left\|\int_{s}^{t} \eta_{\varepsilon}(\tau-\sigma)|\xi|^{\alpha / 2} \widehat{w}(\sigma) d \sigma\right\|_{L^{2}}^{2} d \tau \\
& \quad \leq C \int_{s}^{t}\left\|\Lambda^{\alpha / 2} w\right\|_{L^{2}}^{2} d \tau .
\end{aligned}
$$

Similarly, for $J_{2}, J_{3}$, it follows that

$$
\begin{aligned}
J_{2}+J_{3} & \int_{s}^{t} \int_{\mathbb{R}^{2}} u \cdot \nabla \varphi_{\varepsilon} w d x d \tau \\
& -\int_{s}^{t} \int_{\mathbb{R}^{2}}(\bar{u}-u) \cdot \nabla \varphi_{\varepsilon} \theta d x d \tau \\
\leq & C \int_{s}^{t}\left\|\mathscr{R}^{\perp} \theta\right\|_{L^{4 /(2-\alpha)}}\left\|\nabla \varphi_{\varepsilon}\right\|_{L^{4 / \alpha}}\|w\|_{L^{2}} d \tau \\
& +\int_{s}^{t}\left\|\mathscr{R}^{\perp} w\right\|_{L^{2}}\left\|\nabla \varphi_{\varepsilon}\right\|_{L^{4 / \alpha}}\|\theta\|_{L^{4 /(2-\alpha)}} d \tau \\
\leq & C \int_{s}^{t}\left\|\Lambda^{\alpha / 2} \theta\right\|_{L^{2}}\left\|\nabla \varphi_{\varepsilon}\right\|_{L^{4 / \alpha}}\|w\|_{L^{2}} d \tau \\
\leq & C\left(\sup _{0<\tau<\infty}\|w\|_{L^{2}}\right)\left(\int_{s}^{t}\left\|\Lambda^{\alpha / 2} \theta\right\|_{L^{2}}^{2} d \tau\right)^{1 / 2} \\
& \times\left(\int_{s}^{t}\left\|\nabla \varphi_{\varepsilon}\right\|_{L^{4 / \alpha}}^{2} d \tau\right)^{1 / 2}
\end{aligned}
$$




$$
\begin{aligned}
& \leq E_{0}^{1 / 2}\left(\int_{s}^{t}\left\|\Lambda^{\alpha / 2} \theta\right\|_{L^{2}}^{2} d \tau\right)^{1 / 2}\left(\int_{s}^{t}\left\|\Lambda^{\alpha / 2} w\right\|_{L^{2}}^{2} d \tau\right)^{1 / 2} \\
& \leq E_{0}^{1 / 2} \int_{s}^{t}\left\|\Lambda^{\alpha / 2} \theta\right\|_{L^{2}}^{2} d \tau+E_{0}^{1 / 2} \int_{s}^{t}\left\|\Lambda^{\alpha / 2} w\right\|_{L^{2}}^{2} d \tau .
\end{aligned}
$$

Plugging the estimates of $J_{i}(i=1,2,3)$ into (47) yields

$$
|J| \leq E_{0}^{1 / 2} \int_{s}^{t}\left\|\Lambda^{\alpha / 2} \theta\right\|_{L^{2}}^{2} d \tau+E_{0}^{1 / 2} \int_{s}^{t}\left\|\Lambda^{\alpha / 2} w\right\|_{L^{2}}^{2} d \tau \text {. }
$$

In order to estimate $K$, thanks to

$$
\begin{aligned}
& \int_{s}^{t}\left\|\varphi_{\varepsilon}(\tau)\right\|_{H^{\alpha / 2}}^{2} d \tau \\
& \leq C \int_{s}^{t}\left\||\xi|^{\alpha / 2} \widehat{\varphi}_{\varepsilon}(\tau)\right\|_{L^{2}}^{2} d \tau \\
& \leq C \int_{s}^{t} \| \int_{s}^{t} \eta_{\varepsilon}(\tau-\sigma)\left(1+|\xi|^{2}\right)^{-(3-\alpha) / 2} \\
& \leq C \int_{s}^{t}\left\|\int_{s}^{t} \eta_{\varepsilon}(\tau-\sigma)|\xi|^{\alpha / 2} \widehat{w}(\sigma) d \sigma\right\|_{L^{2}}^{2} d \tau(2 t-\tau-\sigma)|\xi|^{\alpha}|\xi|^{\alpha / 2} \widehat{w}(\sigma) d \sigma \|_{L^{2}}^{2} d \tau \\
& \leq C \int_{s}^{t}\left\|\Lambda^{\alpha / 2} w\right\|_{L^{2}}^{2} d \tau
\end{aligned}
$$

together with the Hölder inequality and the Young inequality gives that

$$
\begin{aligned}
K & =-\int_{s}^{t}\left(g, \varphi_{\varepsilon}\right) d \tau \leq \int_{s}^{t}\|g\|_{H^{-\alpha / 2}}\left\|\varphi_{\varepsilon}\right\|_{H^{\alpha / 2}} d \tau \\
& \leq C \int_{S}^{t}\|g\|_{H^{-\alpha / 2}}^{2} d \tau+C \int_{S}^{t}\left\|\Lambda^{\alpha / 2} w\right\|_{L^{2}}^{2} d \tau .
\end{aligned}
$$

Inserting (46), (51), and (53) into (33), we obtain

$$
\begin{aligned}
\left\|\left(1+\Lambda^{2}\right)^{-(3-\alpha) / 4} w(t)\right\|_{L^{2}}^{2} & \\
& -\left\|e^{-\kappa(t-s) \Lambda^{\alpha}}\left(1+\Lambda^{2}\right)^{-(3-\alpha) / 4} w(s)\right\|_{L^{2}}^{2} \\
\leq & |J|+|K| \\
\leq & E_{0}^{1 / 2} \int_{s}^{t}\left\|\Lambda^{\alpha / 2} \theta\right\|_{L^{2}}^{2} d \tau+\left(C+E_{0}^{1 / 2}\right) \int_{s}^{t}\left\|\Lambda^{\alpha / 2} w\right\|_{L^{2}}^{2} d \tau \\
& +C \int_{s}^{t}\|g\|_{H^{-\alpha / 2}}^{2} d \tau
\end{aligned}
$$

which completes the proof of Lemma 8.

\section{Proof of Theorem 2}

With the aid of the global estimates and average decay of the difference $w=\bar{\theta}-\theta$ between the original surface quasigeostrophic equation (1) and the perturbed surface quasigeostrophic equation, the proof of stability is straight forward.
The integration of (28) with respect to $s$ on the interval $((t / 2), t)$ gives

$$
\begin{aligned}
\|w(t)\|_{L^{2}}^{2} \leq & \frac{2}{t} \int_{t / 2}^{t}\|w(s)\|_{L^{2}}^{2} d s \\
& +\frac{C}{t} \int_{t / 2}^{t} \int_{s}^{t}\|\nabla \theta\|_{\mathrm{BMO}} d \tau d s .
\end{aligned}
$$

From the average decay property of $w$ in Section 3, the first term of the right hand side of the above inequality tends to zero as $t \rightarrow \infty$. For the second term of the right hand side, we have

$$
\begin{aligned}
& \frac{1}{t} \int_{t / 2}^{t} \int_{s}^{t}\|\nabla \theta\|_{\mathrm{BMO}} d \tau d s \\
& \quad \leq \frac{C}{t} \int_{t / 2}^{t}\left(\tau-\frac{t}{2}\right)\|\nabla \theta\|_{\mathrm{BMO}} d \tau \\
& \quad \leq C \int_{t / 2}^{t}\|\nabla \theta\|_{\mathrm{BMO}} d \tau,
\end{aligned}
$$

according to the regular condition on $\theta$ in Theorem 2, the second term also tends to zero as $t \rightarrow \infty$. Hence, we derive the following stability property:

$$
\|w(t)\|_{L^{2}} \longrightarrow 0 \quad \text { as } t \longrightarrow \infty
$$

which completes the proof of Theorem 2 .

\section{Acknowledgments}

The authors would like to express their sincere thanks to Professor D. Córdoba for the valuable comments and suggestions. This work is partially supported by the NNSF of China (11271019) and the NSF of Anhui Province (11040606M02), and is also financed by the 211 Project of Anhui University (KJTD002B, KJJQ005).

\section{References}

[1] J. Pedlosky, Geophysical Fluid Dynamics, Springer, New York, NY, USA, 1987.

[2] P. Constantin, A. J. Majda, and E. Tabak, "Formation of strong fronts in the $n$-D quasigeostrophic thermal active scalar," Nonlinearity, vol. 7, no. 6, pp. 1495-1533, 1994.

[3] A. J. Majda and E. G. Tabak, "A two-dimensional model for quasigeostrophic flow: comparison with the two-dimensional Euler flow," Physica D, vol. 98, no. 2-4, pp. 515-522, 1996.

[4] R. Temam, Navier-Stokes Equations. Theory and Numerical Analysis, North-Holland Publishing, Amsterdam, The Netherlands, 1977.

[5] P. Constantin and J. Wu, "Behavior of solutions of 2D quasigeostrophic equations," SIAM Journal on Mathematical Analysis, vol. 30, no. 5, pp. 937-948, 1999.

[6] A. Kiselev, F. Nazarov, and A. Volberg, "Global well-posedness for the critical 2D dissipative quasi-geostrophic equation," Inventiones Mathematicae, vol. 167, no. 3, pp. 445-453, 2007.

[7] L. A. Caffarelli and A. Vasseur, "Drift diffusion equations with fractional diffusion and the quasi-geostrophic equation," Annals of Mathematics, vol. 171, no. 3, pp. 1903-1930, 2010. 
[8] A. Castro and D. Córdoba, "Infinite energy solutions of the surface quasi-geostrophic equation," Advances in Mathematics, vol. 225, no. 4, pp. 1820-1829, 2010.

[9] Q. Chen, C. Miao, and Z. Zhang, "A new Bernstein's inequality and the 2D dissipative quasi-geostrophic equation," Communications in Mathematical Physics, vol. 271, no. 3, pp. 821-838, 2007.

[10] A. Córdoba and D. Córdoba, "A maximum principle applied to quasi-geostrophic equations," Communications in Mathematical Physics, vol. 249, no. 3, pp. 511-528, 2004.

[11] P. Constantin and J. Wu, "Hölder continuity of solutions of supercritical dissipative hydrodynamic transport equations," Annales de l'Institut Henri Poincaré (C), vol. 26, no. 1, pp. 159$180,2009$.

[12] B.-Q. Dong and Z.-M. Chen, "On the weak-strong uniqueness of the dissipative surface quasi-geostrophic equation," Nonlinearity, vol. 25, no. 5, pp. 1513-1524, 2012.

[13] H. Dong and N. Pavlović, "Regularity criteria for the dissipative quasi-geostrophic equations in Hölder spaces," Communications in Mathematical Physics, vol. 290, no. 3, pp. 801-812, 2009.

[14] N. Ju, "Existence and uniqueness of the solution to the dissipative 2D quasi-geostrophic equations in the Sobolev space," Communications in Mathematical Physics, vol. 251, no. 2, pp. 365-376, 2004.

[15] H. Wang and Z. Zhang, "A frequency localized maximum principle applied to the 2D quasi-geostrophic equation," Communications in Mathematical Physics, vol. 301, no. 1, pp. 105-129, 2011.

[16] J. Wu, "Global solutions of the 2D dissipative quasi-geostrophic equation in Besov spaces," SIAM Journal on Mathematical Analysis, vol. 36, no. 3, pp. 1014-1030, 2005.

[17] D. Chae, P. Constantin, and J. Wu, "Inviscid models generalizing the two-dimensional Euler and the surface quasi-geostrophic equations," Archive for Rational Mechanics and Analysis, vol. 202, no. 1, pp. 35-62, 2011.

[18] D. Chae, P. Constantin, D. Córdoba, F. Gancedo, and J. Wu, "Generalized surface quasi-geostrophic equations with singular velocities," Communications on Pure and Applied Mathematics, vol. 65, no. 8, pp. 1037-1066, 2012.

[19] C. Fefferman and J. L. Rodrigo, "Analytic sharp fronts for the surface quasi-geostrophic equation," Communications in Mathematical Physics, vol. 303, no. 1, pp. 261-288, 2011.

[20] B. Khouider and E. S. Titi, "An inviscid regularization for the surface quasi-geostrophic equation," Communications on Pure and Applied Mathematics, vol. 61, no. 10, pp. 1331-1346, 2008.

[21] C. Miao and L. Xue, "Global well-posedness for a modified critical dissipative quasi-geostrophic equation," Journal of Differential Equations, vol. 252, no. 1, pp. 792-818, 2012.

[22] B.-Q. Dong and Q. Liu, "Asymptotic profile of solutions to the two-dimensional dissipative quasi-geostrophic equation," Science China. Mathematics, vol. 53, no. 10, pp. 2733-2748, 2010.

[23] C. J. Niche and M. E. Schonbek, "Decay of weak solutions to the 2D dissipative quasi-geostrophic equation," Communications in Mathematical Physics, vol. 276, no. 1, pp. 93-115, 2007.

[24] Y. Zhou, "Decay rate of higher order derivatives for solutions to the 2-D dissipative quasi-geostrophic flows," Discrete and Continuous Dynamical Systems, vol. 14, no. 3, pp. 525-532, 2006.

[25] Y. Zhou, "Asymptotic behaviour of the solutions to the 2D dissipative quasi-geostrophic flows," Nonlinearity, vol. 21, no. 9, pp. 2061-2071, 2008.
[26] D. Chae and J. Lee, "Global well-posedness in the super-critical dissipative quasi-geostrophic equations," Communications in Mathematical Physics, vol. 233, no. 2, pp. 297-311, 2003.

[27] Z.-M. Chen and W. G. Price, "Stability and instability analyses of the dissipative quasi-geostrophic equation," Nonlinearity, vol. 21, no. 4, pp. 765-782, 2008.

[28] B.-Q. Dong and Z.-M. Chen, "Asymptotic stability of the critical and super-critical dissipative quasi-geostrophic equation," Nonlinearity, vol. 19, no. 12, pp. 2919-2928, 2006.

[29] B.-Q. Dong and Z.-M. Chen, "A remark on regularity criterion for the dissipative quasi-geostrophic equations," Journal of Mathematical Analysis and Applications, vol. 329, no. 2, pp. 12121217, 2007.

[30] H. Kozono, "Asymptotic stability of large solutions with large perturbation to the Navier-Stokes equations," Journal of Functional Analysis, vol. 176, no. 2, pp. 153-197, 2000.

[31] L. Zhang, "New results of general $n$-dimensional incompressible Navier-Stokes equations," Journal of Differential Equations, vol. 245, no. 11, pp. 3470-3502, 2008.

[32] Y. Zhou, "Asymptotic stability for the 3D Navier-Stokes equations," Communications in Partial Differential Equations, vol. 30, no. 1-3, pp. 323-333, 2005.

[33] F. Marchand, "Weak-strong uniqueness criteria for the critical quasi-geostrophic equation," Physica D, vol. 237, no. 10-12, pp. 1346-1351, 2008.

[34] A. Pazy, Semigroups of Linear Operators and Applications to Partial Differential Equations, vol. 44 of Applied Mathematical Sciences, Springer, New York, NY, USA, 1983. 


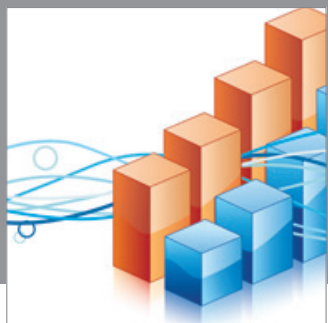

Advances in

Operations Research

mansans

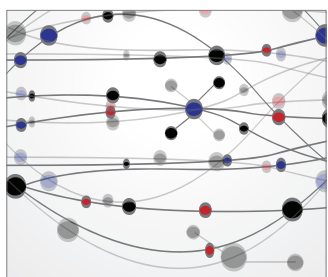

The Scientific World Journal
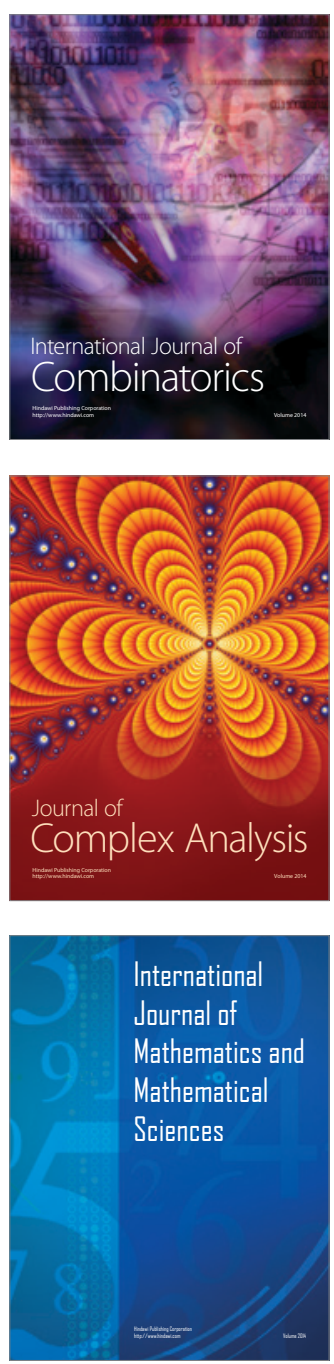
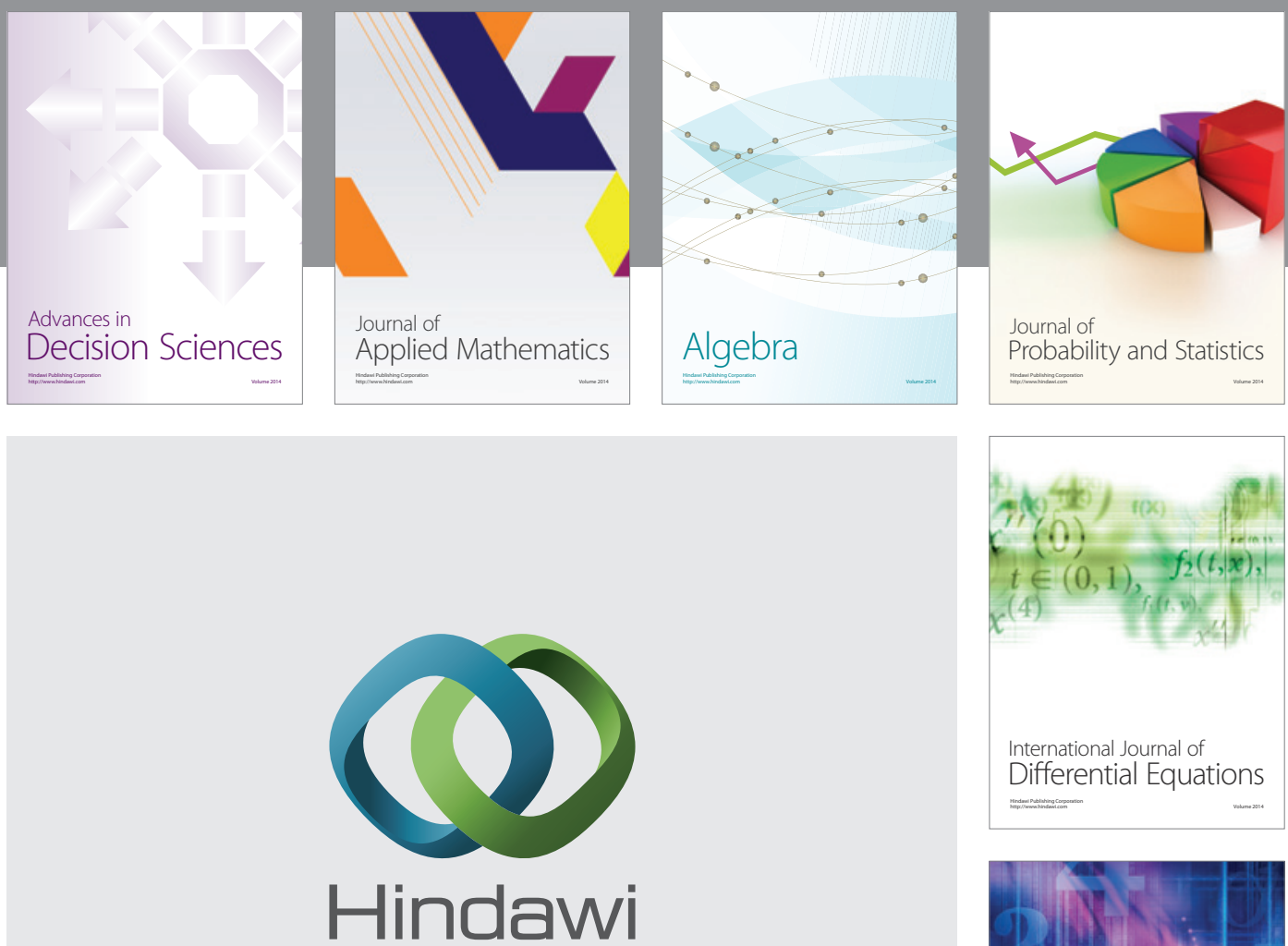

Submit your manuscripts at http://www.hindawi.com
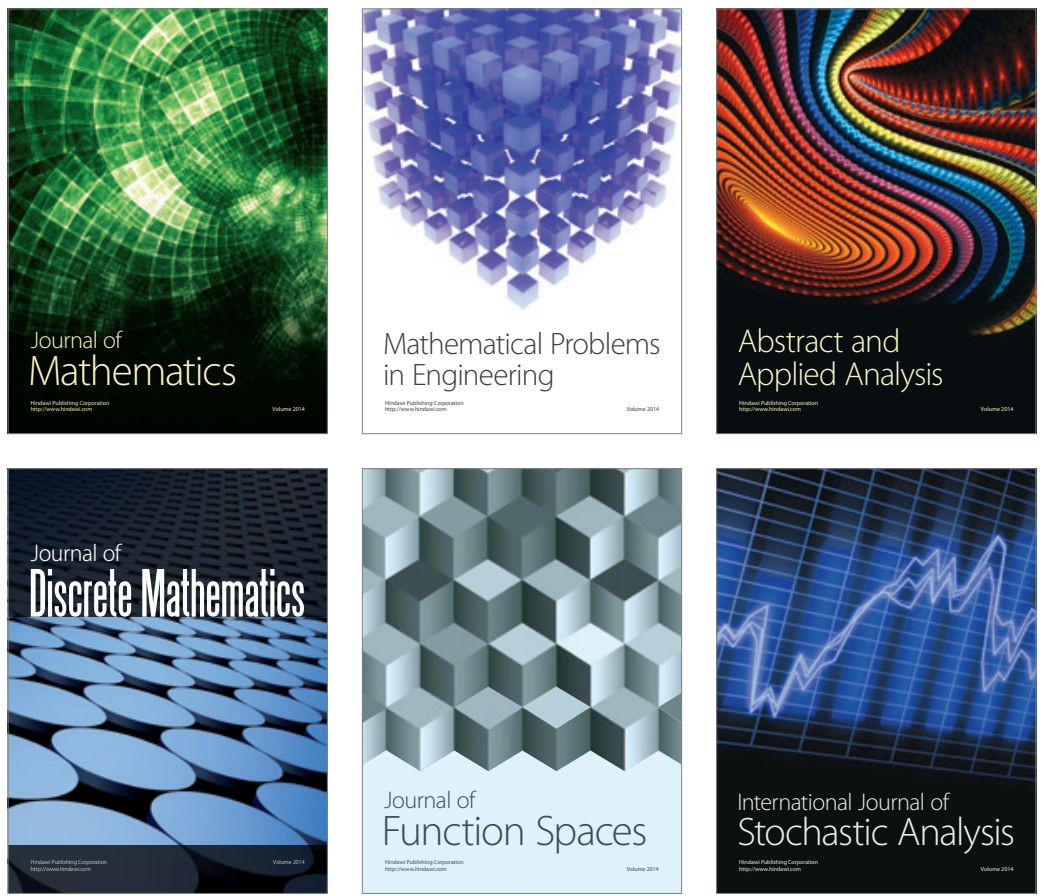

Journal of

Function Spaces

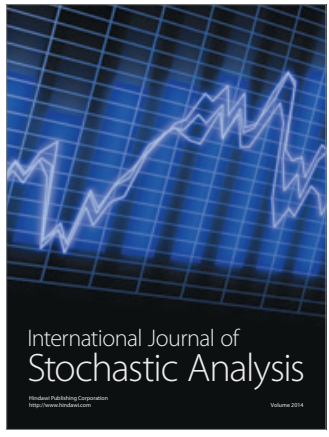

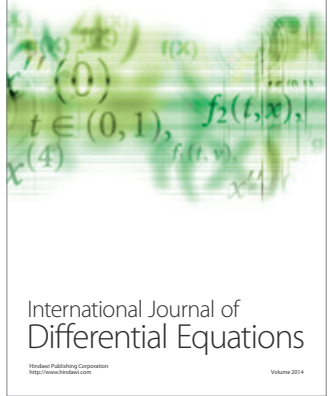
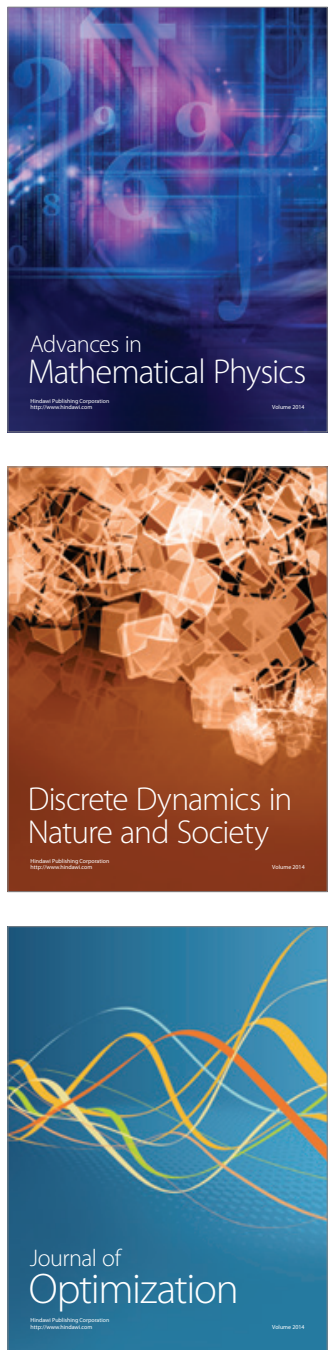